\title{
Polarization measurements on a magnetic quadrupole line in Ne-like barium
}

\author{
E. Takács, ${ }^{*}$ E. S. Meyer, J. D. Gillaspy, and J. R. Roberts \\ Atomic Physics Division, National Institute of Standards and Technology, Gaithersburg, Maryland 20899 \\ C. T. Chantler, ${ }^{\dagger}$ L. T. Hudson, and R. D. Deslattes \\ Quantum Metrology Division, National Institute of Standards and Technology, Gaithersburg, Maryland 20899 \\ C. M. Brown and J. M. Laming \\ E. O. Hulburt Center for Space Research, Naval Research Laboratory, Washington, D.C. 20375 \\ J. Dubau \\ Observatoire de Paris, CNRS UPR No. 176, 92195 Meudon Cedex, France \\ M. K. Inal \\ Institut des Sciences Exactes, Departement de Physique, Boite Postale 119, 13000 Tlemcen, Algeria
}

(Received 12 December 1995)

\begin{abstract}
We have measured the absolute polarization of the $2 p^{6}{ }^{1} S_{0}-2 p^{5}\left({ }^{2} P_{3 / 2}^{o}\right) 3 s[3 / 2]_{2}^{o}$ magnetic quadrupole transition in Ne-like barium, excited in an electron-beam ion trap at a variety of energies. We find strong evidence for the existence of resonant excitation processes that are not explained by our collisional-radiative calculations even when the polarization arising from impact excitation is included. At energies well away from where the resonances occur, the agreement between experiment and theory is good. [S1050-2947(96)06208-7]

PACS number(s): 32.10.-f, 32.30.Rj, 34.80.Kw
\end{abstract}

\section{INTRODUCTION}

In the absence of strong external electric or magnetic fields, atomic states differing in magnetic quantum number, but otherwise having identical principal and angular momentum quantum numbers, are degenerate in energy. Since the magnetic quantum numbers describe the spatial orientation of the atom's electron charge cloud, there may still be observable differences between such degenerate states if some type of spatial asymmetry is present. For example, if collisional excitation occurs by impact in a preferred direction, the magnetic sublevels of the excited state can be populated with nonstatistical probabilities. When the state decays, the emitted electromagnetic radiation will be spatially anisotropic and partially polarized [1].

Anisotropic excitation mechanisms are quite common in astrophysical plasmas and are readily reproduced in a laboratory environment. In solar flares, ions and atoms can be excited by electrons moving along fixed magnetic field lines, which give rise to a preferred direction in space [2]. A similar situation occurs in supernova shock waves [3] and also in polar aurorae and possibly in jets in active galactic nuclei. On earth, there have been many crossed-beam or beam-foil studies whereby atoms and ions are excited in a spatially asymmetric way [4].

In the experiment described in this paper, we study the

\footnotetext{
*Also at Experimental Physics Department, Kossuth University, Debrecen, Bem t. 18/a., Hungary.

Present address: School of Physics, University of Melbourne, Parkville, Victoria 3052, Australia.

${ }^{\ddagger}$ Also at SFA Inc., Landover, MD 20785.
}

polarization of radiation emitted from ions that have been excited by impact with a unidirectional monoenergetic electron beam inside an electron-beam ion trap (EBIT). The EBIT is a powerful tool for creating very highly charged ions for atomic structure and electron-ion interaction studies. Techniques for measuring electron-impact ionization [5], excitation [6], and recombination [7] cross sections using an EBIT have been demonstrated; however, these measurements have all been adjusted to take into account polarization effects.

Inside an EBIT, the ions interact with a narrow (about 60$\mu$ m-diam) beam of electrons. This well-collimated electron beam acts as a quantization axis, creating a cylindrically symmetric environment for the ions. Care must be taken then in interpreting emission line intensities when they are used for obtaining electron-ion interaction cross sections. Polarization of the emitted radiation is especially important when measurements are made with spectrometers in which the energy disperser is polarization selective (e.g., Bragg crystal $\mathrm{x}$-ray spectrometers). Even when a polarization-insensitive energy detector is used [e.g., solid-state $\mathrm{Si}(\mathrm{Li})$ detector], polarization is important because the detector is generally positioned normal to the electron beam rather than at the "magic angle" of $55^{\circ}$ (i.e., the angle $\theta$ at which polarization for dipole radiation disappears, given by $\cos ^{2} \theta=1 / 3$ ). Depending upon the experiment, however, polarization can be a tool rather than a complication in the analysis. The measurement of the polarization or the angular distribution of photon emission gives information about the magnetic sublevels involved in electron-ion collisions that would normally remain hidden in a simple energy dispersive measurement. As we will illustrate below, polarization-sensitive measurements may also be used to detect resonance processes that would 
otherwise be too weak to be observed directly. In what follows, the polarization $P$ is defined as

$$
P=\frac{I_{\|}\left(90^{\circ}\right)-I_{\perp}\left(90^{\circ}\right)}{I_{\|}\left(90^{\circ}\right)+I_{\perp}\left(90^{\circ}\right)},
$$

where $I_{\|}\left(90^{\circ}\right)$ and $I_{\perp}\left(90^{\circ}\right)$ are the intensities of the parallel and perpendicularly polarized radiation measured at $90^{\circ}$ with respect to the axis of symmetry (the electron-beam direction in our experiment).

\section{OBSERVED Ne-LIKE M2 TRANSITION}

In the standard spectroscopic notation for $J_{1} l$ (pair) coupling [8] used for noble-gas spectra, the spectral line we report on in this paper arises from the $2 p^{6}{ }^{1} S_{0}-2 p^{5}\left({ }^{2} P_{3 / 2}^{o}\right) 3 s[3 / 2]_{2}^{o}$ magnetic quadrupole transition in Ne-like barium (hereinafter referred to as the $M 2$ transition or the $M 2$ line). This transition was originally observed in Ne-like iron created in the solar corona [9]. It has since been observed in several other Ne-like systems in a laboratory Tokamak source [10], as well as in an EBIT [11]. In barium, the energy of this transition is calculated to be $4.563 \mathrm{keV}$, corresponding to a wavelength of $2.717 \AA$. The upper level is the lowest-energy excited state of the Ne-like systems. Because of its high angular momentum, many of the high angular momentum excited states preferentially decay to it. This results in a complicated cascade feeding scheme.

Because the transition is magnetic quadrupole, it has a relatively small Einstein- $A$ coefficient for an x-ray transition: about $3.0 \times 10^{8} \mathrm{~s}^{-1}$ in Ne-like barium [13]. However, in low-density plasmas such as that in an EBIT, the relative intensity of the line is comparable to that of the large Einstein- $A$ coefficient lines. This is because of the strong cascade feeding and the fact that the number of decays per unit time is bottlenecked by the electron-impact excitation rates, rather than the decay rates.

In previous EBIT work, the $M 2$ line was studied by Beiersdorfer et al. [11] using a flat crystal spectrometer. The electron-beam energy dependence of the intensity relative to a reference line made it possible to separately measure the effects of different indirect line formation mechanisms. These included resonance excitation of the line, dielectronic recombination onto F-like barium, and inner-shell ionization of Na-like barium. As the authors pointed out in their paper, their diffractive crystal was oriented to preferably reflect $\mathrm{x}$ rays with polarization vector parallel to the electron beam direction. They could not orient it for $\mathrm{x}$ rays of complementary polarization and therefore had to use theoretical estimates of the polarization to compare their observed line intensities with predictions based on the various line formation mechanisms (both direct and indirect). They estimated the polarization $P$, defined in Eq. (1) above, to be $-0.05 \pm 0.10$ over the entire range of electron-beam energies used. That is, they assumed that the polarization had no dependence upon electron-beam energy and justified this assumption with calculations of the impact energy dependence (or, rather, relative independence) of the polarization of the $2 p^{6}{ }^{1} S_{0}-2 p^{5}\left({ }^{2} P_{3 / 2}^{o}\right) 3 d[5 / 2]_{1}^{o}$ electric-dipole line [11] using the method of Zhang, Sampson, and Clark [12]. However, as we remarked above, the formation of the upper level of the M2 line is dominated by cascade decays from high angular momentum states. The relative contribution of the different cascade paths dramatically changes with electron-beam energy. Some of the paths are completely excluded once the energy of the electron beam falls below the excitation threshold for the root state of the path (i.e., the highest-energy state in the path). In turn, the feeding of the different magnetic sublevels of the $M 2$ line also changes with the electron-beam energy. Thus, even if the direct electron-impact excitation cross sections for the magnetic sublevels do not change relative to one another with impact energy, there may still be a significant change in the polarization because of the (energydependent) cascade feeding process. Studying the importance of this effect on the energy dependence of the polarization was the primary motivation for the present work.

\section{CAlCulations}

To gain a better understanding of the cascade feeding of the upper level of the $M 2$ line we carried out calculations with a collisional-radiative model. An analysis using the HULLAC computer code [13] was done at a number of electron-impact energies between 5.2 and $7.8 \mathrm{keV}$, which coincided with the energy range in our experiment. The output of the code gives the relative populations of the levels, the decay rates, electron-impact excitation rates, and energies for the different transitions involved. The calculation included all of the $n=3$ and $n=4$ energy levels of Ne-like barium. Because the excitation threshold energies vary a great deal over the $n=3$ and $n=4$ levels, the number of levels involved in the cascade process increases quickly with electron-beam energy. At $5.2 \mathrm{keV}$, only 23 levels can be excited, whereas at $7.8 \mathrm{keV}$, all $89 n=3$ and $n=4$ levels can play some role. If we consider that the population transfer between the different magnetic sublevels strongly depends on the angular momentum values of the upper and lower levels of the cascading transition, it is clear that the change in the relative population of the magnetic sublevels of the M2 line can be significant. Figure 1 shows the Grotrian diagram of the levels involved in the population of the upper level of the $M 2$ line at $7.8 \mathrm{keV}$ beam energy. It can be seen from this figure that the upper level is preferentially populated from upper states with total angular momentum different from zero. The various excitation and cascade fractions were calculated using the HULLAC code.

The cross section and collisional-radiative programs in the HULLAC code do not treat sublevels of different $M$ for a given $J$. In order to model the polarization expected following cascades through a number of levels, we wrote a collisional-radiative program that explicitly considers each $M$ sublevel. In order to simplify the problem somewhat, we only considered collisional excitations and deexcitations between the ground state and the various excited levels, since $\mathrm{X}$-ray radiative transitions are much faster than collisional transitions in the EBIT. Using angular momentum relationships, the Einstein- $A$ coefficient between magnetic sublevels in terms of the $M$ averaged $A$ coefficient $A\left(J_{i} \rightarrow J_{j}\right)$ is [14] 


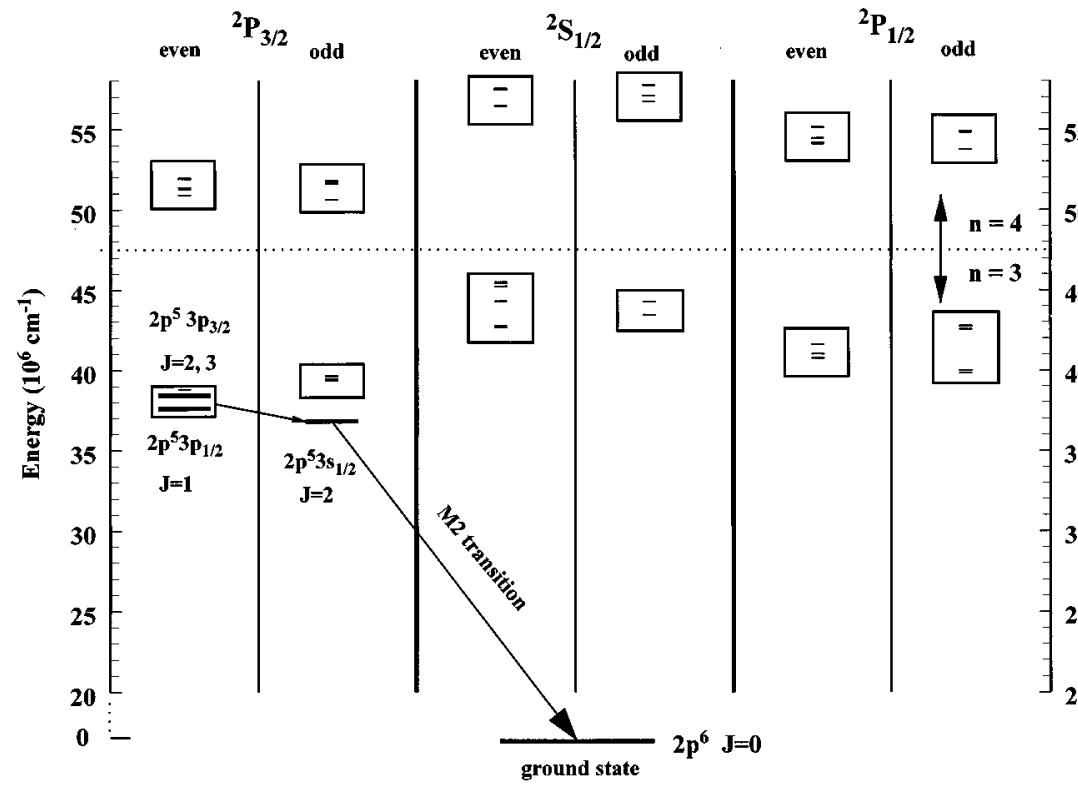

FIG. 1. Partial Grotian diagram showing all the levels involved in the cascade feeding of the upper level of the $M 2$ transition at $7.81 \mathrm{keV}$ beam energy. The dominant levels are shown with long bars and connected with arrows, while the levels that individually contribute less than a few percent to the population are shown by short bars.

$$
\begin{aligned}
A\left(J_{i}, M_{i} \rightarrow\right. & \left.J_{j}, M_{j}\right)=\left|\left(\begin{array}{lll}
J_{j} & q & J_{i} \\
-M_{j} & m & M_{i}
\end{array}\right)\right|^{2} \\
& \times\left(2 J_{i}+1\right) A\left(J_{i} \rightarrow J_{j}\right)
\end{aligned}
$$

where the quantity $\left(\begin{array}{lll}J_{j} & q & J_{i} \\ -M_{j} & m & M_{i}\end{array}\right)$ is the usual Wigner 3- $j$ symbol. In this symbol, $q$ is the multipolarity of the transition and $m=M_{j}-M_{i}$, by the properties of the 3-j symbol. In our modeling, we take the decay rates $A\left(J_{i} \rightarrow J_{j}\right)$ from the previously described HULLAC runs. We take collisional excitation cross sections from the same source, but split these up into the $M$-dependent quantities according to a variety of approximations. The collisional-radiative matrix inversion is run to establish the populations of each $M$ sublevel. Following this, the polarization in each emission line is calculated according to Eq. (19) of Inal and Dubau [15].

As an initial estimate for the polarization fraction of each level, we took the limits given by Percival and Seaton [1]. We assumed that only the orbital angular momentum sublevel $M_{l}=0$ is populated in the electron impact and then coupled the orbital angular momentum with the electron spins to form $J$. As such, this approximation assumes exact $L S$ coupling and therefore a polarization fraction that can only be realized for impact excitation of neutral atoms at threshold. This approximation turns out to overestimate the measured polarization by a large factor, but the dependence on electron-beam energy is qualitatively correct. A similar $j j$ coupling approximation (i.e., assuming sublevels with $M_{l}=0$ only are populated) gives an even larger polarization (leading to a larger discrepancy). $j j$ coupling might be expected to be a better approximation for Ne-like barium, so it is probable that the cause of the discrepancy is our neglect of the depolarization that occurs when the incident electron scatters through a large angle in the Coulomb field of the target ion. The direction of the scattered electron sets the quantization axis for the excited ion, so the stronger the interaction (and thus the larger the scattering angle), the greater the depolarization will be.
Accordingly, we decided to perform detailed calculations of the impact-excitation cross sections from the ground state to the magnetic sublevels of each of the participating excited levels at an impact energy of $410 \mathrm{Ry}(5.578 \mathrm{keV})$. These were undertaken by two of us (J.D. and M.K.I.) essentially following the formalism in Inal and Dubau [15]. The important simplifications are that the collisions are treated nonrelativistically and that we have only one energy point at which the polarization is calculated. Note that the total cross sections we use still retain their energy dependence (which is actually quite weak over the energy range we consider) since these are taken from our earlier HULLAC computations. Eventually a total of 37 levels were included in the calculation, allowing us to employ the cascade model for electron-beam energies up to $5.98 \mathrm{keV}$; above this, higher excited states are involved for which we have no polarization fraction calculations. Putting these results into our modified collisionalradiative model gives the polarizations that are shown in Table I. These results are about a factor of 4 smaller than those predicted by the simple model described in the preceding paragraph.

We should emphasize that only excitation by direct electron impact followed by radiative decay has been included in our model. Since the polarization fractions were calculated at one impact energy only, we are implicitly assuming that the polarization fractions for a given excited state do not change substantially with beam energy over the range of interest. Energy scaling is included for the total cross sections, but not for the fractions going to each $M$ sublevel. Overall, the only substantial omission in our calculation is that resonant processes appearing at energies near 5.2 and $5.8 \mathrm{keV}$ have been neglected. We believe that the effect of resonance excitation is quite evident in the data presented below and can explain the regions in which there is a significant discrepancy between our calculations and our experiment.

\section{INSTRUMENTS}

Descriptions of the history and operating principle of the EBIT have been published elsewhere $[16,17]$. Our machine 
TABLE I. Polarizations of the $M 2$ and $E 1$ lines. Polarizations of the $M 2$ and $E 1$ lines are calculated using the collisional-radiative model and realistic starting polarization fractions. From left to right the columns are beam energy in $\mathrm{keV}, M 2$ line polarization, $E 1$ line polarization, and the number of levels included in the calculation.

\begin{tabular}{lccc}
\hline \hline $\begin{array}{l}\text { Energy } \\
(\mathrm{keV})\end{array}$ & $\begin{array}{c}M 2 \\
(\%)\end{array}$ & $\begin{array}{c}E 1 \\
(\%)\end{array}$ & No. of levels \\
\hline 5.04 & -12.5 & 4.77 & 19 \\
5.10 & -12.5 & 4.77 & 19 \\
5.20 & -12.2 & 3.77 & 21 \\
5.27 & -12.0 & 3.71 & 23 \\
5.40 & -12.1 & 3.57 & 25 \\
5.50 & -12.4 & 2.63 & 33 \\
5.58 & -12.4 & 2.63 & 33 \\
5.70 & -12.4 & 2.63 & 33 \\
5.80 & -14.8 & 2.64 & 37 \\
5.90 & -15.0 & 3.07 & 37 \\
5.98 & -15.0 & 3.07 & 37 \\
\hline \hline
\end{tabular}

[18] is similar in design to the Lawrence Livermore National Laboratory EBIT, which is described in detail in Ref. [19]. The highly charged ions are created, excited, and trapped radially by a $60-\mu \mathrm{m}$-diam, $3500-\mathrm{A} \mathrm{cm}^{-2}$ electron beam. A series of three cylindrical drift tubes-two end cap tubes biased at $250 \mathrm{~V}$ positive with respect to a center drift tubeprovide axial trapping for the ions. The voltage applied to the center drift tube $V_{0}$ determines the electron-beam energy (5.0-8.0 keV in our experiment). The electron-beam energy is not precisely equal to $e V_{0}$, however, because the space charge of the electron beam itself depresses the on-axis potential. We corrected for this effect using a simple calculation based on Gauss's law. Our electron-beam energy scale has perhaps a \pm 50 -eV absolute uncertainty due to the spacecharge correction; the relative uncertainty is on the order of only a few $\mathrm{eV}$, though. Observation of $\mathrm{x}$ rays emitted by ions in the trap is made at $90^{\circ}$ with respect to the electron-beam direction through a series of two beryllium windows that have a total thickness of $0.175 \mathrm{~mm}$. Since barium, which is a dopant in the electron gun cathode, boils off of the cathode and fills the trap automatically, it is one of the easiest elements to study in an EBIT. Just by turning on the electron beam and tuning to an energy above the $3.3-\mathrm{keV}$ ionization potential of Na-like barium, an abundant sample of Ne-like barium is created. About $81 \%$ of naturally occurring barium has zero nuclear spin, so that the effect of the hyperfine interaction on line polarizations is negligible [20].

The measurement was carried out using two identical Johann-type, bent crystal x-ray spectrometers operating simultaneously. A description of the spectrometers and detectors can be found in Ref. [21]. For the wavelength range studied (approximately $2.72 \AA$ ), we used a $\operatorname{Ge}(220)$ $(2 d=4.00 \AA)$ crystal. During the polarization measurement, the two spectrometers were installed so that their respective reflection planes were perpendicular to each other and at $90^{\circ}$ to the electron-beam direction. The Bragg angle is near $45^{\circ}\left(42.8^{\circ}\right)$, so the spectrometers function as near-perfect $\mathrm{x}$ ray polarizers. The polarization of the emitted radiation is obtained by inserting the observed normalized intensities in Eq. (1) and then dividing the result by a correction factor
$Q$ [22], where $Q=1-Y 1-Y 2$, with $Y$ 's representing the amount of parallel-polarized radiation that leaks through into the perpendicular-polarized measurement (and vice versa). The $Q$ factor depends on crystal quality, curvature, alignment, and angle and is very close to unity for high-quality crystals and a Bragg angle close to $45^{\circ}$. A very thin perfect crystal (or a thick or thin mosaic crystal) would have $Y=X /(1+X)$, where $X=\cos ^{2}(2 \theta)$ or about $0.6 \%$ for the exact angle used in our experiment. For the case of a thick perfect crystal, the exponent on the cosine would be 1 and the value of $X$ would be $7.7 \%$. Our crystal has been previously quantified and is intermediate in thickness between the limiting forms, leading to $Y$ values that are about $1.9 \%$ and an overall value of $Q=0.963(5)$.

\section{DETERMINATION OF THE ABSOLUTE POLARIZATION}

In order to determine the absolute line polarization, the orthogonal spectrometers had to be intensity cross calibrated. This can be done by observing an unpolarized line if one can be found near the same energy as the line under study. This method of intensity calibration takes account of the different geometrical and detector efficiency factors at the same time. The ideal candidate isotropic line is one that has a $J=0$ upper level, since in that case there is only one magnetic sublevel and therefore $P=0$. For intensity cross calibration, we used the $2 p^{6}{ }^{1} S_{0}-2 p^{5}\left({ }^{2} P_{3 / 2}^{o}\right) 3 s[3 / 2]_{1}^{o}$ electric dipole transition in Ne-like barium (hereinafter referred to as the $E 1$ transition or the $E 1$ line). This line appears at $4.568 \mathrm{keV}$, which is much closer to the $M 2$ line than a similar electric dipole transition that was used for the same purpose by Beiersdorfer et al. [11]. The proximity of our $E 1$ line to the M2 line allowed us to simultaneously observe them in high resolution with our spectrometers.

Calculations using the collisional-radiative model and the HULLAC code (including all $n=3$ and $n=4$ states) predict that at an electron-beam energy of $7.8 \mathrm{keV}$, the upper level of the $E 1$ line is primarily ( $>80 \%$ ) populated by cascades from states with $J=0$ angular momentum. As a consequence, even though the upper level of the $E 1$ line has nonzero angular momentum, it is mostly unpolarized and isotropic. Figure 2 shows the partial Grotrian diagram of the levels involved in the population of the upper level of the $E 1$ line, which we constructed from the output of the HULLAC code. Using the HULLAC data, we estimate that the absolute value of the polarization for the $E 1$ line is less than $3 \%$ over the entire range of beam energies in our experiment. For calibration purposes, we assumed complete isotropy for the $E 1$ line and took account of the small polarization due to $J \neq 0$ cascades in the error bars on our final results for the $M 2$ line polarization.

It is interesting to compare the ratio of the efficiencies of the two spectrometers based upon observation of the mostly unpolarized $E 1$ line with a sophisticated computer model. The two spectrometers are very nearly identical, but because the $\mathrm{x}$-ray source is in the shape of a thin cylinder (only ions within the $60 \mu \mathrm{m}$ diameter of the electron beam and within the $2 \mathrm{~cm}$ length of the center drift tube can be excited within the EBIT), there is a rather large geometrical effect; that is, their relative efficiencies depend strongly upon their orienta- 


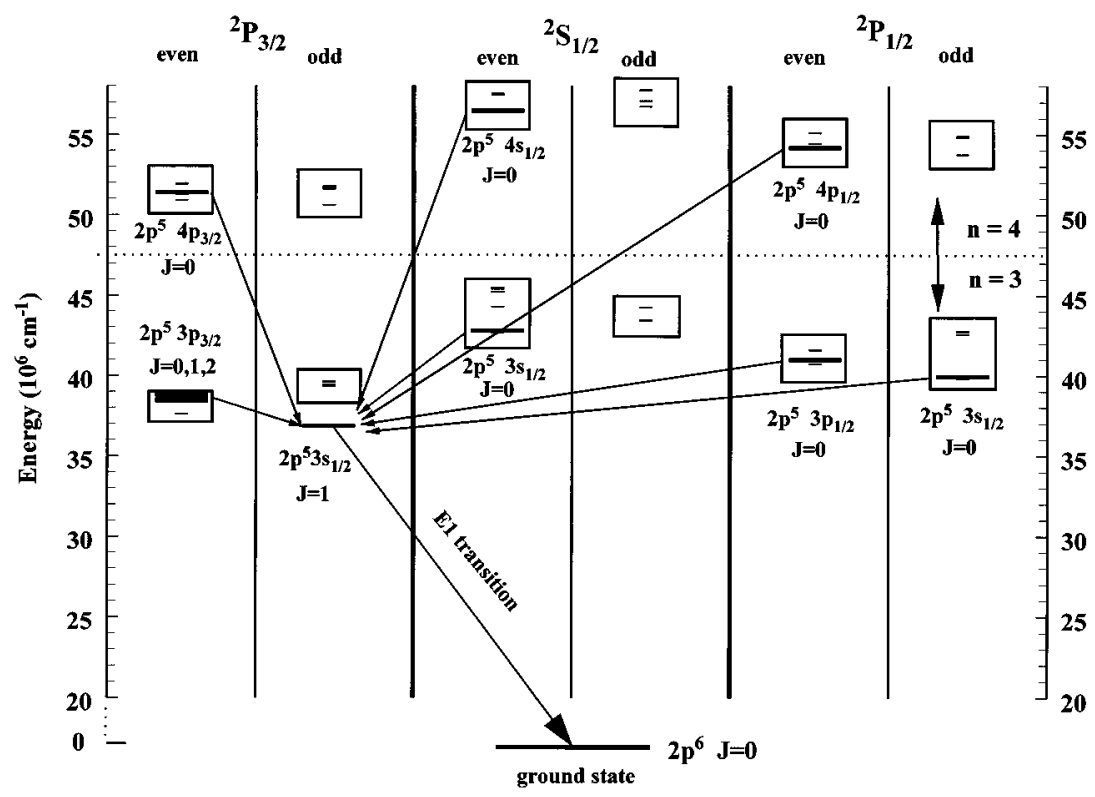

FIG. 2. Partial Grotrian diagram showing all the levels involved in the calculation of the cascade feeding of the upper level of the $E 1$ transition for electron-beam energy $7.81 \mathrm{keV}$. The notation is explained in the caption to Fig. 1.

tion. The computer model [23] predicts that the spectrometer perpendicular to the electron beam has a factor of $1.9 \pm 0.20$ greater efficiency than the one oriented parallel to the electron beam. Using the unpolarized $E 1$ line as a reference, we observe experimentally an efficiency factor of 1.83.

\section{DATA ACQUISITION AND EVALUATION}

Data were taken at 15 different electron impact energies between 4.97 and $7.81 \mathrm{keV}$. The energies were chosen to fall below the excitation thresholds of certain $n=4$ and $n=3$ levels so as to exclude them completely from the cascade feeding routes to the upper level of the $E 1$ line. Each data point represents between 6 and $12 \mathrm{~h}$ of collection time. Longer times were required for the lowest energies because fewer levels are excited that can potentially feed the $M 2$ or $E 1$ lines, thus making the lines appear weaker. Figure 3 shows typical spectra at a beam energy of $6.00 \mathrm{keV}$ for each spectrometer. The $M 2$ and $E 1$ lines are clearly resolved and no other strong features are present in the spectrum. For all of the data points, electron-beam currents ranged between 135 and $150 \mathrm{~mA}$, depending on the energy we had set. The current and energy were held fixed during the entire collection time and a small amount of $\mathrm{N}_{2}$ was injected to improve evaporative cooling of the trapped barium ions [24]. After the data were taken, the six best spectra from each spectrometer were summed to generate two spectra with the highest signal-to-noise ratio. These were used to determine the line centers as accurately as possible and to extract the individual spectrometer response functions. The line centers and response functions were then held fixed in subsequent fits used to extract the polarization-dependent line intensities. The data evaluation was done using a spectrum fitting program [25]. Initially, peaks were fit to Voigt line shapes, but it was quickly discovered that the peaks had a predominantly Gaussian shape. Accordingly, fits to Gaussian line shapes with linear background subtraction were performed for all of the spectra. From fits to each of the 15 different spectra (representing 15 different electron-beam energies), the intensity of both lines $(J=2$ and $J=1)$ was determined. Compar- ing the intensity ratio of the two lines for each spectrometer gave a measure of the absolute polarization in accordance with Eq. (1).

\section{SYSTEMATIC ERRORS}

We have already mentioned some minor corrections that must be applied to the measured polarization, arising from a small polarization dependence in the crystal reflectivity, as well as a small deviation in the Bragg angle from $45^{\circ}$. Ac-

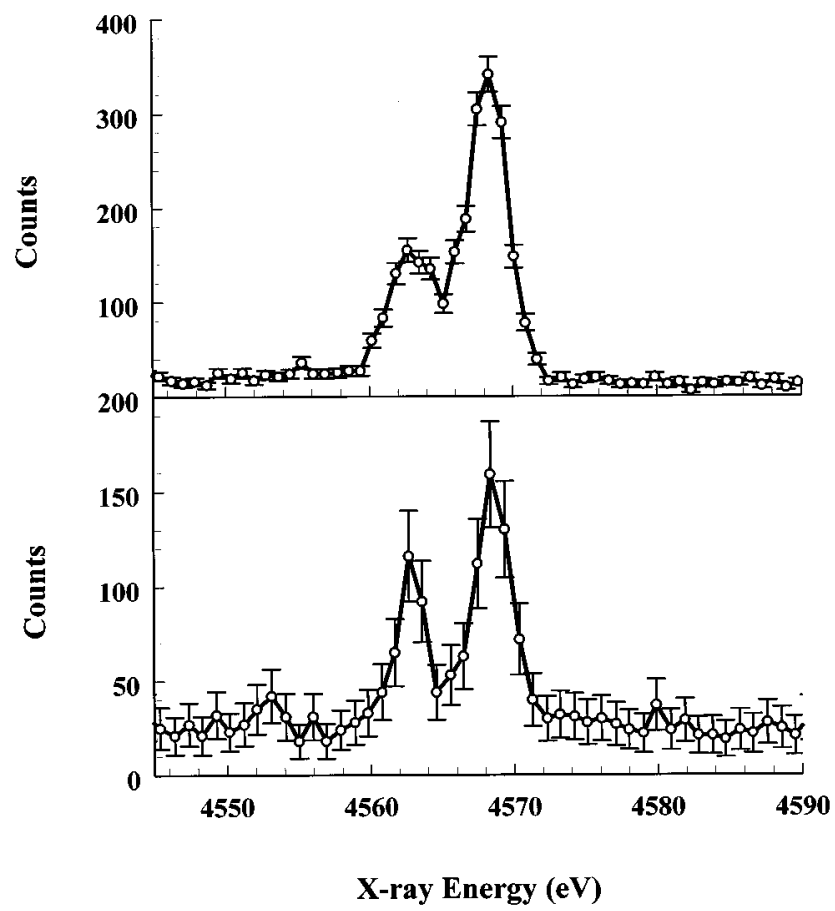

FIG. 3. Simultaneous data from the spectrometers that measure perpendicular (top) and parallel (bottom) polarization. The beam energy was $6.00 \mathrm{keV}$. The $M 2$ and $E 1$ lines are clearly resolved in both spectra. X-ray energy increases to the right, so that the $M 2$ line lies to the left (lower energy) of the $E 1$ line. 
cording to our model calculations [23] of the crystal reflectivity (accurate to $1-2 \%$ ), the corresponding correction to the polarization is no more than $1.6 \%$, with a far smaller uncertainty. These model calculations also allow us to apply a theoretical normalization to the two spectrometers so we can experimentally confirm that the reference line is indeed unpolarized, $P=0.0(1)$. In addition, the model calculations imply that the $\mathrm{x}$-ray signal emerging from our EBIT forms an effective source height of 13(7) $\mathrm{mm}$.

Another correction and accompanying source of error arises from the transverse motion of electrons in the beam. The presence of the strong magnetic field $B_{0}$ in the EBIT allows rigid-rotor motion of the electron beam at a maximum angular frequency approximately equal to the electron cyclotron frequency $\omega_{c e}=e B_{0} / m_{e}$ [26]. This rotation can develop when the beam moves through a magnetic-field gradient. In the EBIT, the electrons move from a region of near-zero magnetic field at the cathode to a region of 3-T magnetic field in the center of the trap [27]. The incident electron velocity vectors therefore lie on the surface of an inverted cone and this leads to some depolarization from the case of a perfectly aligned beam.

The total velocity of the electron beam is determined by the potential $V_{0}$ applied to the center drift tube. We estimate the transverse component of this total velocity using several independent methods. First, we note that under our conditions of 3-T field, the angular frequency is $5 \times 10^{11} \mathrm{~Hz}$, which means a maximum transverse kinetic energy of 700 $\mathrm{eV}$ for electron orbits inside a $60-\mu \mathrm{m}$-diam beam. This transverse energy is consistent with the fact that we have been unable to operate our EBIT below $700 \mathrm{eV}$ total beam energy without picking up significant stray currents in the electrodes (such as the electron gun anode) that surround the electron beam at various points along its trajectory. The maximum possible rotation velocity estimated in this way gives an angle of incidence with respect to normal ("pitch angle") ranging from $21^{\circ}$ to $17^{\circ}$ for total beam energies ranging from 5.0 to $7.8 \mathrm{keV}$, respectively. The final angular velocity of the beam could be less than the maximum, however. It is essentially determined by the magnetic field at the cathode since $v_{\perp}^{2} / B$ is an adiabatic invariant for a charged particle traveling through a fixed magnetic field. Thus we make our second estimate as follows. We note that at the cathode, $v_{\perp}^{2}$ is of order $2 k T_{c} / m_{e}$, where $T_{c}=1500 \mathrm{~K}$ is the temperature of the cathode. The cathode magnetic field $B_{c}$ is not known, but it is believed to be of order a few hundred microtesla (a few gauss) since that is the level of control that the EBIT bucking coil (which is used to null out the field at the cathode) gives. As discussed in the Appendix, a theoretical estimate of the cathode magnetic field gives values $B_{c}=240-210 \mu \mathrm{T}(2.4-2.1 \mathrm{G})$, which give pitch angles of $24^{\circ}-20^{\circ}$ for beam energies in the range $5.0-7.8 \mathrm{keV}$, respectively. This second estimate should be considered as a rough estimate for the "typical" pitch angles, but since it is somewhat larger than the more rigorously obtained pitch angles obtained in the first estimate, we use the first estimate for analysis of our data.

With the incident electron velocity vectors lying on the surface of an inverted cone, there are two geometric effects that will cause some depolarization. The first is that the angle of observation with respect to the incident electron velocity vector will deviate from $90^{\circ}$. The second and more serious problem is that for electron velocity vectors with nonzero components lying in the plane normal to the observation direction, the axes for the polarization measurement will be rotated. The first case was accounted for by running polarization calculations as described above, but for an average angular deviation from observation at right angles. The cosine of this angle is given by

$$
\langle\cos \beta\rangle=(2 / \pi) \int_{0}^{\pi / 2} \sin \left(\phi_{p}\right) \cos (\theta) d \theta=2 \sin \left(\phi_{p}\right) / \pi \simeq 0.2,
$$

where $\phi_{p}$ is the typical electron pitch angle to the beam axis, given by $\arctan \left(v_{\perp} / v_{\|}\right)$, taken here to be about $17^{\circ}$. In the limit of small angles, the observed polarization is reduced by a factor $1-\langle\cos \beta\rangle^{2}[15]$, or about 0.96 .

The second effect reduces the observed polarization by a factor

$$
\begin{aligned}
\langle\cos (2 \phi)\rangle & =(2 / \pi) \int_{0}^{\pi / 2} \frac{1-\tan ^{2}\left(\phi_{p}\right) \sin ^{2}(\theta)}{1+\tan ^{2}\left(\phi_{p}\right) \sin ^{2}(\theta)} d \theta \\
& =1-\tan ^{2}\left(\phi_{p}\right) \\
& \simeq 0.90 .
\end{aligned}
$$

This expression is easily obtained from the general form of Eq. (1) rotated through an angle $\phi$ rather than evaluated at $90^{\circ}$.

The combined result of the two geometric effects is the product of the two, so the measured polarization is approximately $87 \%$ of the true value. This percentage will change slightly (about 5\%) with beam energy in the range we consider, but not sufficiently to alter the energy dependence of our observed polarization.

We do not have direct measurements of the magnitude of the transverse kinetic energy of electrons in the EBIT or of the cathode magnetic field (from which the final transverse kinetic energy can be estimated). We note, however, that a polarization measurement of a suitable line might in fact be a good way of measuring this. A strong line with a large polarization could be monitored as the cathode magnetic field $B_{c}$ is changed through tuning of the bucking coil current.

Another potential source of systematic error arises from anisotropy in the emitted radiation from the $E 1$ line used for normalization. In the analysis, we determined the cascade feeding scheme with the HULLAC code as in the case of the $M 2$ line. Our estimate for the polarization of the $E 1$ line, which is probably an overestimate for energies well above the excitation threshold, is $P=-1.6 \%$ at high energies. Over the entire range of our energies, the $E 1$ polarization is estimated to remain below $3 \%$ and the effect on the polarization of the $M 2$ line, in turn, is less than 0.01 . Note that in estimating the polarization of the $E 1$ line we have neglected the effect of resonances, but these should further drive the polarization towards the assumed value of zero, just as they do for the $M 2$ line.

Finally, there is an uncertainty in the absolute beam energy. As discussed above, there is a depression of the energy due to the negative space charge of the electron beam, which tends to reduce the on-axis potential with respect to the drift 


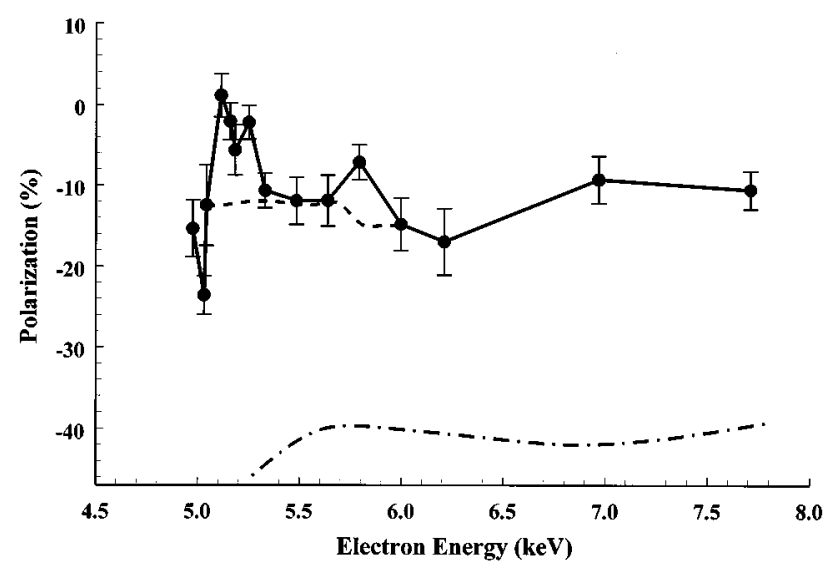

FIG. 4. Polarization $P$ of the $M 2$ line as a function of beam energy. The 15 beam energies were chosen to fall below the excitation thresholds of certain cascading levels, thus completely excluding those levels from the cascade scheme. Both the simple theory (which neglects levels with a nonzero orbital angular momentum, as appropriate near the excitation threshold) and the best presently available theoretical estimate (which includes collisional radiative effects but not resonant excitation processes) are also shown with a dash-dotted line and a dashed line, respectively.

tube electrode voltage $V_{0}$. This space-charge correction can be calculated; however, there is an additional complication because trapped positive ions (both barium ions and background gas ions) partially neutralize the electron-beam space charge. This neutralization is difficult to determine theoretically, but it is possible to estimate it experimentally by observing the wavelength of a radiative recombination line (which changes with electron-beam energy) or by observing the change in intensity of a dielectronic recombination resonance line with beam energy. We determined that the overall space-charge correction in our situation was $250 \mathrm{eV}$, with an uncertainty of $\pm 50 \mathrm{eV}$. The uncertainty, however, represents an unknown but constant offset that must be applied to all of the data; the relative beam energies are known to within $\pm 5 \mathrm{eV}$. Thus, in the results that follow, the energy scale has an offset uncertainty of about $\pm 50 \mathrm{eV}$, but the shape of the curve of polarization versus energy is certain within the onestandard-uncertainty error bars shown.

\section{RESULTS}

Our measurements are shown in Fig. 4 as a plot of the polarization $P$ of the $M 2$ line as a function of electronimpact energy. The beam energies were chosen to exclude certain levels from the cascade scheme, thereby simplifying the theoretical calculations and maximizing the significance of each data point. For example, all $n=4$ levels were excluded in the measurements below $6.3 \mathrm{keV}$. Also, at these electron-impact energies, neither the resonance excitation to the F-like charge state nor the inner-shell ionization of the Na-like charge state can play a role since both are energetically excluded.

The theoretical estimates are also shown in Fig. 4. The dash-dotted line corresponds to an approximation where only the sublevels with zero orbital angular momentum are populated. This is a very crude approximation and supposed to be valid only near the excitation threshold.

The fairly strong decrease in the absolute value of the polarization around $5.5 \mathrm{keV}$ beam energy coincides with the intensity increase seen by Beiersdorfer et al. [11] in the parallel polarized component of the $M 2$ line. This intensity increase was interpreted by Beiersdorfer et al. [11] to be the result of a resonance excitation of the upper level of the $M 2$ line. In this two-step process, a dielectronic resonance transition to one of the autoionizing Na-like levels takes place. The dielectronic process is followed by an autoionization, whereby the final state includes the upper level of the $M 2$ line. If the interpretation is correct, the $25 \%$ decrease in the line polarization could be accounted for by the resonance excitation process itself. However, since polarization calculations already show a similar tendency, we have to conclude that at least part of the polarization change is due to the change in the cascade feeding scheme for the upper level of the $M 2$ line. The assumption made by Beiersdorfer et al. in Ref. [11] of a $-5 \pm 10 \%$ polarization that is independent of electron-impact energy holds over a good part of the energy range of our measurement, but not in those places where resonances can occur. In our case, we measured a negative line polarization with an average value of $-0.12 \pm 0.10 \%$. The absolute value of the polarization increases close to the direct excitation threshold energy and decreases strongly near 5.2 and $5.8 \mathrm{keV}$, where $L N O$ and $L O O$ dielectronic resonance (DR) excitations are allowed (in Auger notation, $L O O$ denotes an $L$-shell electron promoted to the $O$ shell while an incident unbound electron is captured in the $O$ shell). These results do not change the basic conclusions of Ref. [11] and we believe that these deviations of the polarization from its average value are in fact due to the indirect processes reported by Beiersdorfer et al. [11]. In fact, we attribute the region of strong deviation near $5.8 \mathrm{keV}$ to an $L O O$ resonance process that was not observed in Ref. [11].

It appears that the resonant excitation process (DR followed by autoionization) creates unpolarized radiation. In a simple picture, this might be expected, since even if the initial DR process populates the $m$ sublevels of the doubly excited intermediate state nonuniformly, the subsequent electron emission in the autoionization process will scramble the quantization axis of the final-state singly excited ion.

\section{CONCLUSION}

We have measured the absolute polarization of the $2 p^{6}{ }^{1} S_{0}-2 p^{5}\left({ }^{2} P_{3 / 2}^{o}\right) 3 s[3 / 2]_{2}^{o}$ magnetic quadrupole transition in Ne-like barium. We find that for electron-impact excitation in the energy range from 5.0 to $7.8 \mathrm{keV}$, this line shows a strong negative polarization. The polarization typically falls between $-4 \%$ and $-18 \%$, but changes sharply near $5.0 \mathrm{keV}$, as well as near $5.1-5.3$ and $5.8 \mathrm{keV}$.

We have also found a steady decrease in the polarization between 5.5 and $6.0 \mathrm{keV}$, which should be due at least in part to the change in the cascade feeding routes. It appears that the quantitative agreement between models and experiment is greatly improved when realistically calculated polarization fractions are included. The precise degree of agreement at certain energies is probably fortuitous, however, since important resonant processes have not been included. In fact, it seems that polarization measurement may represent a sensi- 
tive method for confirming the presence of such resonant excitation processes. In our data, for example, a decrease in the absolute value of the polarization is quite prominent near $5.8 \mathrm{keV}$, which we interpret as being due to an $L O O$ dielectronic resonance excitation. This resonance is difficult to isolate in a plot of total line intensity versus beam energy and was not observable in Ref. [11].

Finally, we note that polarization measurements might also be used to determine the magnitude of rigid-rotor motion in the electron beam of an EBIT. This rotation might be controlled through tuning of the cathode magnetic field, and it would be interesting to see if such effects are indeed important in EBIT.

\section{ACKNOWLEDGMENTS}

We thank E. Bell and F.G. Serpa for assistance during the course of this experiment and V. Jacobs for helpful discussions. One of the authors (E.T.) acknowledges the Foundation for Hungarian Higher Education and Research for support.

\section{APPENDIX}

Here we discuss the intensity of the magnetic field at the EBIT cathode $B_{c}$. While the minimum electron-beam radius is obtained for zero field at the cathode, under this condition the electrons cannot enter the trap. By maximizing an expression for the current density in the ion trap $j$,

$$
j=n_{e} q v_{\|}=\frac{N_{e}}{\pi R^{2}} q\left(v^{2}-v_{\perp t}^{2}\right)^{1 / 2}
$$

in the trap with respect to $B_{c}$, where $v_{\perp t}$ is the perpendicular velocity in the trap (as opposed to at the cathode, denoted by $\left.v_{\perp c}\right), n_{e}$ is the electron density, and $N_{e}$ is the number of electrons per unit length in the beam (assumed constant), we can derive a theoretical estimate for the optimum cathode magnetic field. The beam radius $R$ is given by Herrmann's theory [28]

$$
R=r_{b}\left\{\frac{1}{2}+\frac{1}{2}\left[1+4\left(\frac{8 k T_{c}}{m_{e}} \frac{r_{c}^{2}}{\eta^{2} B_{t}^{2} r_{b}^{4}}+\frac{B_{c}^{2} r_{c}^{4}}{B_{t}^{2} r_{b}^{4}}\right)\right]^{1 / 2}\right\}^{1 / 2},
$$

where $r_{b}$ is the Brillouin radius, $r_{c}$ is the cathode radius, $B_{t}$ is the trap magnetic field, $T_{c}$ is the cathode temperature, $m_{e}$ is the electron mass, and $\eta$ is the charge to mass ratio $q / m_{e}$ of the electron. A consideration of adiabatic invariant quantities leads to the following expression for the perpendicular velocity in the trap:

$$
v_{\perp t}=v_{\perp c}\left(\frac{B}{B_{c}}\right)^{1 / 2}
$$

Substituting Eqs. (A2) and (A3) into (A1), differentiating with respect to $B_{c}$, setting the result equal to zero, and rearranging gives

$$
B_{c}^{3}=B_{t}^{3} \frac{v_{\perp c}^{2}}{v^{2}-v_{\perp c}^{2} B_{t} / B_{c}} \frac{R^{4}-R^{2} r_{b}^{2} / 2}{2 r_{c}^{4}}
$$

which can be simplified for $v \gg v_{\perp t}$ and $R \simeq r_{b}$ to give

$$
B_{c} \simeq B_{t}\left(\frac{v_{\perp c}^{2}}{4 v^{2}} \frac{R^{4}}{r_{c}^{4}}\right)^{1 / 3}
$$

Taking $T_{c}=1500 \mathrm{~K}$, which gives $v_{\perp c} \simeq 1.5 \times 10^{5} \mathrm{~ms}^{-1}$, $R=3 \times 10^{-5} \mathrm{~m}, r_{c}=1.5 \times 10^{-3} \mathrm{~m}$, and $B_{t}=3 \mathrm{~T}$, gives a value for $B_{c}$ of $(2.1-2.4) \times 10^{-4} \mathrm{~T}$ for electron-beam energies of 5.0-7.8 keV, in reasonable agreement with (and completely independent from) the value inferred from the sensitivity of the electron beam to the bucking coil current.
[1] I. C. Percival and M. J. Seaton, Philos. Trans. R. Soc. London Ser. A 251, 113 (1958).

[2] See, e.g., J. M. Laming, Astrophys. J. 357, 275 (1990).

[3] J. M. Laming, Astrophys. J. 362, 219 (1990).

[4] J. M. Laming, Nucl. Instrum. Methods Phys. Res. Sect. B 43, 359 (1989).

[5] D. Vogel, P. Beiersdorfer, R. Marrs, K. Wong, and R. Zasadzinski, Z. Phys. D 21, S193 (1991); K. L. Wong, P. Beiersdorfer, D. Vogel, R. Marrs, and M. Levine, ibid. 21, S197 (1991).

[6] R. E. Marrs, M. A. Levine, D. A. Knapp, and J. R. Henderson, Phys. Rev. Lett. 60, 1715 (1988); C. M. Brown, U. Feldman, G. A. Doschek, J. F. Seely, R. E. LaVilla, V. L. Jacobs, J. R. Henderson, D. A. Knapp, R. E. Marrs, P. Beiersdorfer, and M. A. Levine, Phys. Rev. A 40, 4089 (1989).

[7] D. A. Knapp, R. E. Marrs, M. A. Levine, C. L. Bennett, M. H. Chen, J. R. Henderson, M. B. Schneider, and J. H. Scofield, Phys. Rev. Lett. 62, 2104 (1989); D. A. Knapp, Z. Phys. D 21, S143 (1991).

[8] W. C. Martin, R. Zalubas, and L. Hagan, Atomic Energy Levels-The Rare-Earth Elements, Natl. Bur. Stand. (U.S.)
Circ. No. 60 (U.S. GPO, Washington, DC, 1978).

[9] M. Loulergue and H. Nussbaumer, Astron. Astrophys. 24, 209 (1973); J. H. Parkinson, ibid. 24, 215 (1973).

[10] M. Klapisch et al., Phys. Lett. 69A, 34 (1978).

[11] P. Beiersdorfer, A. L. Osterheld, M. H. Chen, J. R. Henderson, D. A. Knapp, M. A. Levine, R. E. Marrs, K. J. Reed, M. B. Schneider, and D. A. Vogel, Phys. Rev. Lett. 65, 1995 (1990).

[12] H. L. Zhang, D. H. Sampson, and R. E. H. Clark, Phys. Rev. A 41, 198 (1990).

[13] M. Klapisch, A. Bar Shalom, W. H. Goldstein, E. Meroz, A. Chon, and M. Cohen, HullaC code for atomic physics, 1988 (unpublished).

[14] This is derived from the Wigner-Eckart theorem and properties of the $3-j$ symbols.

[15] M. K. Inal and J. Dubau, J. Phys. B 20, 4221 (1987); 22, 3329 (1989) (this paper corrects some typographical errors in the relevant equation).

[16] R. E. Marrs, P. Beiersdorfer, and D. Schneider, Phys. Today 47 (10), 27 (1994).

[17] J. D. Gillaspy, Proceedings of the Sixth International Sympo- 
sium on Electron Beam Ion Sources [Phys. Scr. (to be published)].

[18] J. D. Gillaspy, J. R. Roberts, C. M. Brown, and U. Feldman, in VIth International Conference on the Physics of Highly Charged Ions, edited by P. Richard, M. Stöckli, C. L. Cocke, and C. D. Lin, AIP Conf. Proc. No. 274 (AIP, New York, 1993), p. 682.

[19] M. A. Levine, R. E. Marrs, J. R. Henderson, D. A. Knapp, and M. B. Schneider, Phys. Scr. T22, 157 (1988).

[20] J. R. Henderson, P. Beiersdorfer, C. L. Bennett, S. Chantrenne, D. A. Knapp, R. E. Marrs, M. B. Schneider, K. L. Wong, G. A. Doschek, J. F. Seely, C. M. Brown, R. E. LaVilla, J. Dubau, and M. A. Levine, Phys. Rev. Lett. 65, 705 (1990).

[21] B. P. Duval, J. Barth, R. D. Deslattes, A. Henins, and G. G. Luther, Nucl. Instrum. Methods 222, 274 (1984); A. Henins, Rev. Sci. Instrum. 58, 1173 (1987).
[22] B. Cleff, Acta Phys. Pol. A 61, 285 (1982).

[23] C. T. Chantler (private communication) [using the computer code described in C. T. Chantler, Ph.D. thesis, Oxford University, 1990 (unpublished)]; J. Appl. Crystallogr. 25, 674 (1992); 25, 694 (1992)].

[24] B. M. Penetrante, J. N. Bardsley, M. A. Levine, D. A. Knapp, and R. E. Marrs, Phys. Rev. A 43, 4873 (1991).

[25] J. Végh, Candidate's thesis, Institute of Nuclear Research of the Hungarian Academy of Sciences (ATOMKI), 1991 (unpublished).

[26] R. C. Davidson, Theory of Nonneutral Plasmas (Benjamin, New York, 1974).

[27] J. D. Jackson, Classical Electrodynamics (Wiley, New York, 1975).

[28] G. Herrmann, J. Appl. Phys. 29, 127 (1958). 